\title{
Mycophagous growth of Collimonas bacteria in natural soils, impact on fungal biomass turnover and interactions with mycophagous Trichoderma fungi
}

\author{
Sachie Höppener-Ogawa ${ }^{1}$, Johan HJ Leveau ${ }^{1}$, Johannes A van Veen ${ }^{1,2}$ and Wietse De Boer ${ }^{1}$ \\ ${ }^{1}$ Centre for Terrestrial Ecology, Netherlands Institute of Ecology (NIOO-KNAW), Heteren, The Netherlands \\ and ${ }^{2}$ Institute of Biology Leiden, Leiden University, Leiden, The Netherlands
}

\begin{abstract}
Bacteria of the genus Collimonas are widely distributed in soils, although at low densities. In the laboratory, they were shown to be mycophagous, that is, they are able to grow at the expense of living hyphae. However, so far the importance of mycophagy for growth and survival of collimonads in natural soil habitats is unknown. Using a Collimonas-specific real-time PCR assay, we show here that the invasion of field soils by fungal hyphae (Absidia sp.) resulted in a short-term, significant increase (average fourfold) of indigenous collimonads. No such responses were observed for other soil bacteria studied (Pseudomonas, Burkholderia, PCR-denaturing gradient gel electrophoresis patterns of total bacteria and Burkholderia). Hence, it appears that the stimulation of growth of Collimonas bacteria by fungal hyphae is not common among other soil bacteria. In the same field soils, Trichoderma, a fungal genus known for mycophagous (mycoparasitic) growth, increased upon introduction of Absidia hyphae. Hence, mycophagous growth by Collimonas and Trichoderma can occur in the same soils. However, in controlled experiments (sand microcosms), collimonads appeared to have a negative effect on mycophagous growth of a Trichoderma strain. The effect of mycophagous growth of collimonads on fungal biomass dynamics was studied in sand microcosms using the same Absidia sp. as a test fungus. The growth of collimonads did not cause a significant reduction in the Absidia biomass. Overall, the study indicates that mycophagous nutrition may be important for collimonads in natural soils, but the impact on fungal biomass turnover is likely to be minor.
\end{abstract}

The ISME Journal (2009) 3, 190-198; doi:10.1038/ismej.2008.97; published online 16 October 2008

Subject Category: microbe-microbe and microbe-host interactions

Keywords: bacterial mycophagy; Collimonas; Trichoderma; fungal biomass turnover

\section{Introduction}

Mycophagy, that is, the feeding on living fungi, has been reported for soil bacteria of the genus Collimonas (De Boer et al., 2004; Leveau and Preston, 2008). Mycophagous growth was based on the proliferation of collimonads in gnotobiotic sand microcosms that contained living fungal mycelium as the only source of energy and carbon (De Boer et al., 2001). However, whereas the nutrient-poor conditions in these microcosms were realistic for natural soils, other conditions, for example, the absence of other (micro) organisms and plant roots, were different from the natural soil environment.

Correspondence: W De Boer, Plant Microorganism Interactions, Netherlands institute of Ecology, Boterhoeksestraat 48, Heteren, 6666 GA, The Netherlands.

E-mail: w.deboer@nioo.knaw.nl

Received 22 June 2008; revised 8 September 2008; accepted 8 September 2008; published online 16 October 2008
In a field inventory, we investigated the distribution of collimonads among different soils (Höppener-Ogawa et al., 2007). Collimonads appeared to be more abundant in fungal-rich natural grassland and forest soils than in fungal-poor arable soils. Yet, no clear relationship between fungal biomass and abundance of collimonads was observed. Hence, further studies are required to elucidate the importance of mycophagy for in situ growth of collimonads, in particular because they can grow on a wide range of organic substrates, that is, they are facultative mycophagous (De Boer et al., 1998).

Unlike the few studies that have been carried out on bacterial mycophagy, fungal mycophagy, which is better known as mycoparasitism, has been the subject of many studies (Lorito et al., 1998; Harman, 2006). Most of these studies deal with Trichoderma species (Elad et al., 1983; Donzelli and Harman, 2001). In particular, the application of Trichoderma spp. to control plant pathogenic fungi (for example, 
Rhizoctonia spp. and Fusarium spp.) has received much attention (Hermosa et al., 2000; Whipps, 2001; Harman, 2006). Trichoderma spp. are widely distributed in the terrestrial environment (Samuels, 1996). However, as for collimonads, the actual importance of mycophagous growth for Trichoderma spp. under natural soil conditions is not known. Like collimonads, Trichoderma spp. are facultative mycophagous (De Boer et al., 1998). Hence, for both collimonads and Trichoderma spp., other sources of energy, for example, soil organic matter and root exudates, may be more important for their growth than fungal-derived substrates.

The current study was aimed (1) to find evidence for the importance of mycophagous growth for collimonads in natural soils, (2) to assess the consequence of mycophagous growth of collimonads for fungal biomass production and (3) to examine possible interactions between collimonads and Trichoderma spp. to perform their mycophagous growth.

\section{Materials and methods}

\section{Soils}

In January 2007, soil samples (upper $10 \mathrm{~cm}$ of mineral layer) were collected from sites where collimonads had been detected earlier (HöppenerOgawa et al., 2007). The soils were a forest soil (site 2), two grassland soils (sites 4 and 5) and a soil from an abandoned arable site (site 22), respectively. Characteristics of these soils (sites 2, 4, 5 and 22) were described earlier as $\mathrm{pH}_{\text {water }} 3.5,4.9,5.3$ and 5.6, total organic carbon $36.1,9.6,77.3$ and $31.1 \mathrm{~g} \mathrm{~kg}^{-1}$ and $\mathrm{C} / \mathrm{N}$ ratio $26.8,11.9,10.2$ and 19.2 , respectively. (Höppener-Ogawa et al., 2007).

\section{Bacterial strains}

The strains used in this study were Collimonas fungivorans Ter 331 (AJ310395) and Ter $6^{\mathrm{T}}$ (LMG 21973), Collimonas arenae Ter $10^{\mathrm{T}}$ (LMG 23964) and Collimonas pratensis Ter $91^{\mathrm{T}}$ (LMG 23965) (De Boer et al., 2004; Höppener-Ogawa et al., 2008). These 4 strains have been isolated from the same soil (De Boer et al., 2004). Hence, their co-occurrence is natural. Based on the experimental conditions and feeding-preferences, the mixture of the four strains was used to provide the best chances for mycophagous growth in this study (De Boer et al., 2004). Pseudomonas fluorescens strain AD21 is a soil isolate that has been described earlier (De Boer et al., 2007). Burkholderia JS is a soil isolate kindly provided by Dr Drigo (Plant Research International, Wageningen, The Netherlands), which has $98 \%$ identity with a Burkholderia cepacia strain (AY741358).

\section{Fungal strains}

On the basis of earlier study, it was evident that the growth of collimonads can be especially stimulated by zygomycetal fungi (De Boer et al., 2001). In the current study, we used a zygomycetal fungus, isolated from a grassland soil, that was identified as Absidia sp. on the basis of the sequence analysis of the internal transcribed spacer (ITS) region in the nuclear ribosomal repeat unit, using primers ITS1-F and ITS4-B (Gardes and Bruns, 1993). Absidia spp. are common saprotrophic soil fungi (Hoffmann et al., 2007). Trichoderma harzianum CECT 2413 (Rubio et al., 2005) was purchased from the Spanish type culture collection (CECT, University of Valencia, Spain).

Experiment 1: growth responses of indigenous Collimonas spp., Pseudomonas spp., Burkholderia spp. and Trichoderma spp. to invasion of field soils by Absidia mycelium

Soils collected from each sampling site were homogenized and portions ( $40 \mathrm{~g}$ ) of the soils were transferred to Petri dishes (diameter, $8.5 \mathrm{~cm}$ ) and spread evenly. A potato dextrose agar (PDA) disk (diameter, $1 \mathrm{~cm}$ ) from the growing margin of $A b s i d i a$ was inverted and placed on an autoclaved metal slide and was centrally placed in the middle of the Petri dish. The metal coin was used to prevent leaching of nutrients from the agar disks into the soil. The Petri dishes were sealed and incubated at $20^{\circ} \mathrm{C}$. After 1 week of incubation, Absidia mycelium covered the whole soil surface. Samples were taken by scraping the soil surface after 0,2 and 3 weeks of incubation. In addition, comparable samples were taken from Petri dishes without invading Absidia hyphae. For all soils, treatments (with and without Absidia) were performed in sixfold.

Soil DNA extracted from the collected soil samples was used for real-time PCR enumerations of collimonads and fungi belonging to the genus Trichoderma. Real-time PCR enumerations of indigenous Pseudomonas and Burkholderia bacteria as well as PCR-denaturing gradient gel electrophoresis (PCR-DGGE) analysis of the bacterial community structure were performed to evaluate the specificity of the growth increase in collimonads upon introduction of fungal mycelium. DNA was extracted from an amount of soil equivalent to $0.25 \mathrm{~g}$ dry soil using the MOBIO kit (MOBIO Laboratories, Solana Beach, CA, USA) according to the manufacturer's instruction, except that soil DNA was finally eluded in $50 \mu \mathrm{l}$ instead of $100 \mu \mathrm{l}$.

Experiment 2: estimation of the mycophagous biomass production by collimonads and the evaluation of interactions between collimonads and T. harzianum upon mycophagous performance in gnotobiotic sand microcosms

Gnotobiotic sand microcosms were used to study the impact of Collimonas spp. on fungal biomass production. Collimonas strains were pre-grown on chitin-yeast agar at $20^{\circ} \mathrm{C}$ for 14 days as described by 
de Boer et al. (2001). Absidia sp. was grown on PDA at $20^{\circ} \mathrm{C}$ for 4 days.

The four different Collimonas strains were mixed by adding equal number of cells of each strain to P-buffer $\left(\mathrm{KH}_{2} \mathrm{PO}_{4}, 1 \mathrm{gl}^{-1}(\mathrm{pH}\right.$ 6.5)). The suspension was mixed into autoclaved, acid-purified beach sand to give a moisture content of $5 \%$ (wt/wt) and a total bacterial density of $10^{4}$ cells per gram sand (based on microscopic counts), which is a common density of collimonads in field soils (HöppenerOgawa et al., 2007). Portions ( $40 \mathrm{~g}$ ) of the incubated sand were transferred to Petri dishes (diameter, $8.5 \mathrm{~cm}$ ) and spread evenly. The Petri dishes were sealed, placed at $20^{\circ} \mathrm{C}$ and pre-incubated for 1 week to allow the microorganisms to adapt to the prevailing conditions. Next, an agar disk (PDA; diameter, $1 \mathrm{~cm}$ ) from the growing margins of Absidia was inverted and placed on an autoclaved metal slide and this was centrally placed in the middle of Petri dish. The Petri dishes were sealed and incubated at $20^{\circ} \mathrm{C}$. After 3 weeks of incubation, sand was removed from the surface covered by hyphae of Absidia and homogenized before the measurement of fungal biomass (ergosterol) and the abundance of collimonads (real-time PCR).

The same experimental setup was used to the study interactions of collimonads with the mycophagous fungus T. harzianum during growth on Absidia hyphae.

Sterile sand with or without collimonads (see above) was inoculated with a spore suspension $\left(10^{4}\right.$ spores per gram sand) of T. harzianum. Conidia had been produced on PDA and were collected and suspended in P-buffer. After vortexing, the suspension was centrifuged at 4000 r.p.m. for 1 min. The supernatant was collected as spore suspension and was mixed into autoclaved, acid-purified beach sand to give a moisture content of $5 \%$ (wt/wt) and a total density of $10^{4}$ spores per gram sand (based on microscopic counts). All treatments were performed in sixfold. Using the prepared microcosms in the same experimental setup with the inoculation of Absidia (see above), Petri dishes were sealed and incubated at $20^{\circ} \mathrm{C}$. After 3 weeks of incubation, sand was removed from the surface covered by hyphae of Absidia and homogenized before the measurement of the abundance of collimonads and T. harzianum (real-time PCR).

\section{Real-time PCR}

Real-time PCR was performed on a Rotor-Gene 3000 (Corbett Research, Sydney, NSW, Australia). All mixes were made using a CAS-1200 pipetting robot (Corbett Research) to reduce variation caused by pipetting errors. Quantification of collimonads in soil DNA samples was performed as described by Höppener-Ogawa et al. (2007).

For quantification of pseudomonads, Burkholderia spp. and Trichoderma spp., the Absolute qPCR SYBRGreen mixture (ABgene, Epsom, UK) was used at a final concentration of $1 \times$ for the real-time reaction. The standard curves for Pseudomonas and Burkholderia. were made from genomic DNA extracted from a pure culture of P. fluorescens strain AD21 and Burkholderia JS (see strain description). The Pseudomonas-specific primer sets used were PSf (5'-GGTCTGAGAGGATGATCAGT- $\left.3^{\prime}\right)$ and PSr (5'-TTAGCTCCACCTCGCGGC-3') (Widmer et al., 1998). The Burkholderia-specific primer sets used were Burk3 (5'-CTGCGAAAGCCGGAT-3') and BurkR (5'-TGCCATACTCTAGCYYGC-3') (Salles et al., 2002).

For quantification of Trichoderma spp., we used a modified protocol of Hagn et al. (2007). The Trichoderma standard curve was made from the DNA extracted from a pure culture of T. harzianum CECT 2413. PCR amplification was performed using the fungal universal primer sets NS1 (5'-GTAGTCAT ATGCTTGTCTC-3') (White, 1990) and TW13 (5'-GG TCCGTGTTTCAAGACG-3') (Taylor and Bruns, 1999).

For quantification of Absidia sp., real-time PCR quantification was performed using universal fungal-specific primers as described elsewhere (Vainio and Hantula, 2000). In addition, measurement of the fungal cell membrane component ergosterol by an alkaline extraction protocol was performed to quantify the biomass of Absidia sp. (De RidderDuine et al., 2006).

\section{PCR-DGGE analysis}

All PCR reactions and DGGE were carried out as described elsewhere (Salles et al., 2002; Yergeau et al., 2007). The primers 968f-GC and 1378r (Heuer et al., 1997), and FR1-GC and FF390r (Vainio and Hantula, 2000) were used to analyze bacterial and fungal communities, respectively. The nested PCR reactions were performed for Burkholderia spp. with primers Burk3 and R1378 (Salles et al., 2002). The products from the first PCR were diluted at 1:1000 and used as the template in the second PCR with primers Burk3-GC and BurkR (Salles et al., 2002).

\section{Statistical analyses}

The banding patterns of DGGE gels were analyzed using the Image Master 1D program (Amersham Bioscience, Roosendaal, The Netherlands). The resulting binary matrices were exported and used in statistical analysis as 'species' presence-absence matrices.

The effect of the introduction of Absidia hyphae, sampling site and the interaction of these two factors on the community structure as analyzed by PCRDGGE was tested by distance-based redundancy analysis (Legendre and Anderson, 1999). Jaccard's coefficient of similarity was calculated and the resulting similarity matrix was exported to Canoco 4.5 as species data for redundancy analysis (Ter Braak and Šmilauer, 2002). Variables to be included in the model were chosen by forward selection at a 
0.05 baseline. The significance of canonical model was tested with 999 permutations. To test the effects of each of the two variables (Absidia invasion and soil origin), the individual variables were recorded using dummy binary variables, of which one was used in Canoco as the only environmental variable in the model and the other as co-variable. To test the interaction, the only variable entered in the model was the interaction between Absidia invasion and soil origin, whereas both individual factors were included (without interaction) as co-variables. The significances of such models were tested with 999 permutations.

All analyses of variance were performed using Statistica 7.0 (StatSoft Inc., Tulsa, OK, USA). For analysis of variance, normal distribution of data was tested with the Shapiro-Wilks test and variance homogeneity by Levene's test. When data failed to satisfy one of the tests, an appropriate transformation was applied (log or square-root transformation). Tukey's honest significant difference (HSD) method modified for unequal sample size (Unequal N HSD in Statistica) $P<0.05$ was used.

\section{Results}

Growth response of indigenous collimonads and Trichoderma spp. to invasion of field soils by Absidia mycelium (experiment 1)

The effect of the introduction of Absidia mycelium on the number of collimonads is shown in Figures $1 \mathrm{a}$ and $\mathrm{b}$. The real-time PCR-based numbers of indigenous collimonads in the soils were $5.0 \times 10^{4}$ per gram soil for sites 2,4 and 5 , and $7.5 \times 10^{5}$ per gram soil for the site 22 . These numbers did not increase during 2 weeks of incubation without the introduction of fungal hyphae. The numbers of collimonads had increased (4.1-fold on average) in all four soils after 2 weeks of invasion by Absidia hyphae. However, with the exception of soil for the site 4 , the stimulating effect of invasion by Absidia hyphae was no longer apparent when the incubation period was 1 week longer (3 weeks in total).

The growth dynamics of two bacterial genera, Pseudomonas and Burkholderia, was examined to have an indication of the specificity of the growth response of collimonads upon introduction of Absidia. No effects of the introduction of Absidia on bacterial numbers within these genera were detected (Figures 1c-f). The numbers of Burkholderia bacteria in the soil site 4 were under detection limit $\left(1.0 \times 10^{3}\right.$ copies per gram soil) after 3 weeks of incubation.

Real-time PCR-based numbers of indigenous fungi belonging to the genus Trichoderma were significantly increased in two soil sites (2 and 5) after 2 weeks of the introduction of Absidia (Figures $1 \mathrm{~g}$ and $\mathrm{h}$ ). In the soil of site 4 , the increase was significant at the level of $P<0.1$. The average increase in real-time
PCR-based numbers in these three soil sites was 26.8-fold. After 3 weeks of incubation, a significant stimulation of the abundance of Trichoderma spp. was only found for the soil site 5 .

Impact of soil colonization by Absidia mycelium on bacterial and fungal community structure (experiment 1)

The effect of invading Absidia mycelium on microbial community structure as assessed by the PCRDGGE in the different soils was analyzed by distance-based RDA (Table 1, Supplementary Table S1). The bacterial community structure, including Burkholderia community structure, appeared to be unchanged, whereas the fungal community structure was significantly influenced by the introduction of Absidia in all the soils that we tested. For all soils, the band corresponding to Absidia was clearly visible in the gel.

Growth response of Absidia to Collimonas mycophagy (experiment 2)

The introduction of Absidia in purified sand containing collimonads resulted in a 5.2-fold increase in the real-time PCR-based numbers of collimonads in the mycelial zone (Figure 2a). The dynamics of the abundance of Absidia was assessed by quantification of $18 \mathrm{~S}$ rRNA gene copies and ergosterol (Figures $2 \mathrm{~b}$ and c, respectively). Results of real-time PCR indicated that 18S rRNA gene copies of the Absidia tended to increase in the presence of collimonads, although the differences between controls and collimonads-containing microcosms were not significant at the 5\% level. The quantities of the fungal membrane component ergosterol did not differ significantly between controls and collimonads-containing microscosms.

\section{Interaction between Collimonas and Trichoderma} mycophagy (experiment 2)

The increase in the real-time PCR-based numbers of the ITS region of Trichoderma rRNA upon introduction of Absidia was significantly lower in the presence of collimonads (Figure 3). The increase in the numbers of collimonads because of the invasion of Absidia hyphae was not significantly affected by the presence of T. harzianum.

\section{Discussion}

The increase in the numbers of indigenous soil collimonads upon introduction of Absidia mycelium indicates that the fungal-induced growth response of collimonads is not restricted to artificial environments (sand microcosms, agar) but can also occur in natural soils. In contrast to collimonads, the numbers of bacteria belonging to genera Pseudomonas and Burkholderia did not increase after the soil 

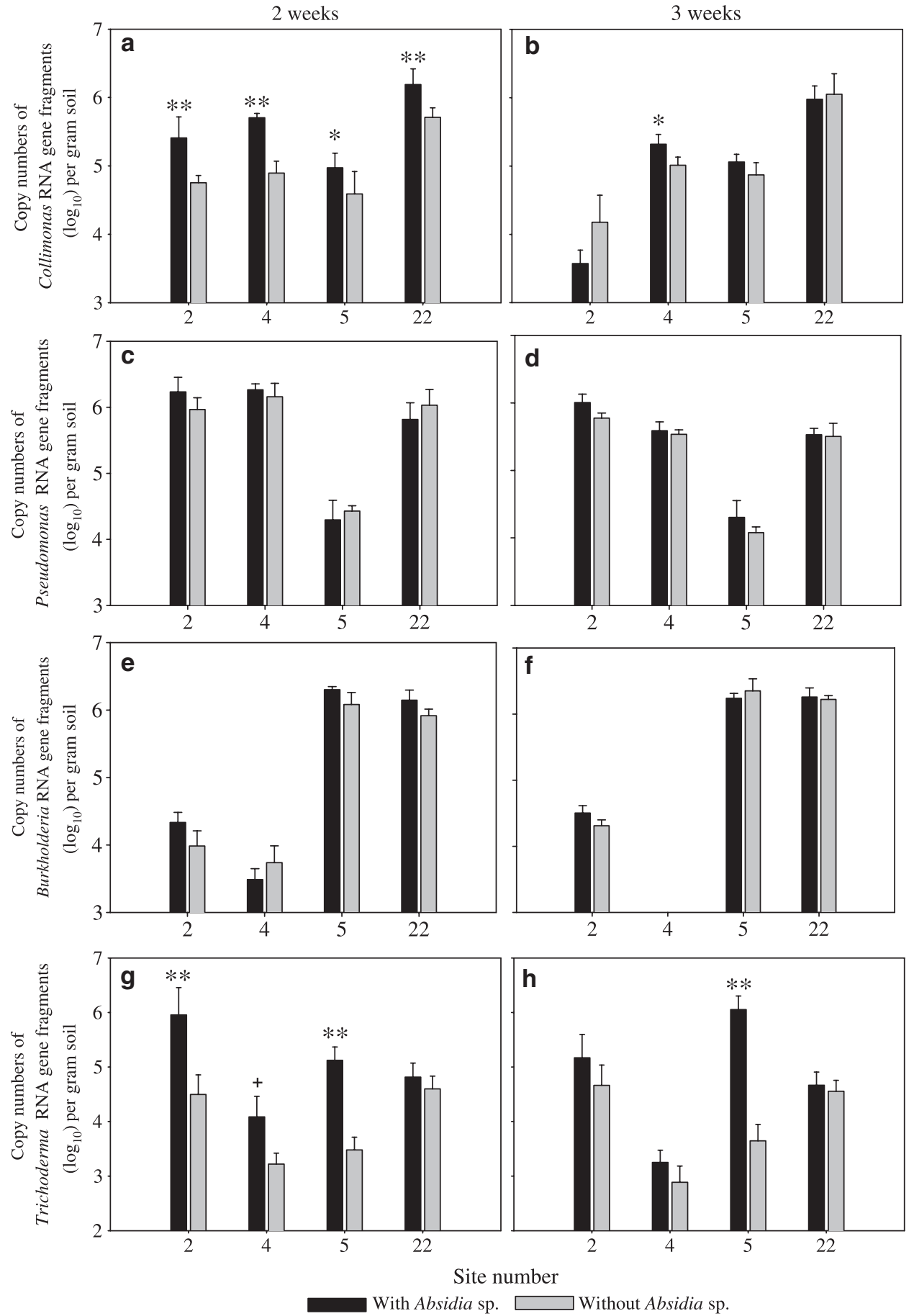

Figure 1 Growth response of indigenous soil bacteria belonging to the genus Collimonas (a and b), Pseudomonas (c and d), Burkholderia (e and f) and of indigenous soil fungi belonging to the genus Trichoderma ( $\mathbf{g}$ and $\mathbf{h}$ ) upon extension of mycelium of the fungus Absidia in four field soil microcosms (sites 2, 5, 4 and 22). Copy numbers of the 16S rRNA of the bacterial genera and of the ITS region of Trichoderma rRNA were determined by real-time PCR in the mycelial zone of Absidia (black bar) and in a comparable zone of microcosms without the introduction of Absidia (gray bar). Real-time PCR-based quantifications were performed 2 and 3 weeks after the introduction of fungal inoculum. Data represent the means and s.d. for six replicates that were harvested at the indicated times. Different symbols within a graph indicate significant differences for a particular soil between microscosms with and without the introduction of Absidia $\left({ }^{+} P \leqslant 0.1,{ }^{*} P \leqslant 0.05\right.$ and $\left.{ }^{*} P \leqslant 0.01\right)$ based on Tukey's HSD test.

had been invaded by Absidia hyphae. Several Pseudomonas and Burkholderia spp. were found to be associated with fungal hyphae, probably growing on fungal exudates (Singh and Arora, 2001; De Boer et al., 2005; Izumi et al., 2008). The fact that the numbers of bacteria belonging to these genera did not increase upon introduction of Absidia hyphae makes it unlikely that the growth response of collimonads was due to the assimilation of exudates. Hence, our investigation points toward 
Table 1 Distance-based redundancy analyses of the effect of invasion of hyphae of the fungus Absidia sp. on microbial community structures (presence-absence of PCR-DGGE bands) in microcosms of four different field soils

\begin{tabular}{|c|c|c|c|c|c|}
\hline \multirow[t]{2}{*}{ Incubation time } & \multirow[t]{2}{*}{ Factors } & \multicolumn{2}{|c|}{ Bacteria } & \multicolumn{2}{|c|}{ Fungi } \\
\hline & & F-ratio & $\mathrm{P}$-value & F-ratio & $\mathrm{P}$-value \\
\hline \multirow[t]{3}{*}{2 weeks } & Absidia & 0.900 & 0.518 & 3.069 & 0.001 \\
\hline & Soil origin & 42.907 & 0.001 & 3.088 & 0.001 \\
\hline & Absidia $\times$ soil origin & 0.900 & 0.464 & 2.629 & 0.001 \\
\hline \multirow[t]{3}{*}{3 weeks } & Absidia & 0.153 & 0.967 & 3.399 & 0.007 \\
\hline & Soil origin & 21.666 & 0.001 & 4.399 & 0.001 \\
\hline & Absidia $\times$ soil origin & 0.071 & 1.000 & 2.728 & 0.001 \\
\hline
\end{tabular}

Abbreviation: PCR-DGGE, PCR-denaturing gradient gel electrophoresis.

an active mycophagous growth by collimonads on Absidia hyphae in field soils.

Comparison of bacterial DGGE patterns between control soils and soils subjected to invasion by Absidia did not reveal other groups of bacteria that were stimulated by the introduction of the fungus. Hence, it appears that the stimulation of Collimonas bacteria growth by fungal hyphae is not common among other soil bacteria. However, the PCR-DGGE analysis does only cover a limited number of dominant bacterial taxa, and the presence of other bacteria with similar growth responses as collimonads can by no means be excluded (Kowalchuk et al., 2004).

Remarkably, the increase of indigenous collimonads upon invasion of Absidia hyphae in the field soils was only short term. Within 3 weeks, numbers had dropped again to the levels of the control for most soils. The same dynamics, that is a short-term increase in collimonads numbers, was also found when Collimonas strains were inoculated in purified sand and exposed to invading hyphae of different fungal species (De Boer et al., 2001; Figure 2a). In the study by De Boer et al. (2001), collimonads increased only during the extension of fungal hyphae and shortly thereafter. The subsequent decrease in Collimonas colony forming units (CFU) in the study by De Boer et al (2001) could have been due to a reduction in cultivability of starving cells. Such an explanation is unlikely for the current study where the real-time PCR enumeration of collimonads was not dependent on the cultivability of strains. As the same dynamics of the numbers of collimonads, that is short-term increase, was found for both the indigenous collimonads in field soils and Collimonas isolates in pure sand, it is unlikely to be caused by predatory or antagonistic soil microorganisms. The same trend in the decrease in real-time PCR-based numbers during prolonged incubation was also found for pseudomonads, Burkholderia spp. and Trichoderma spp. for some of the soils. The increase in the inhibition of real-time PCR in the prolonged incubated samples does not offer an explanation, as we did not find PCR inhibition in any of our samples (data not shown). Hence, for the time being, we cannot explain the apparent decrease in collimonads after the fungal-induced increase.

Although there is a significant growth increase in collimonads to invading hyphae in both field soils and pure sand, the absolute amount of bacterial biomass produced at the expense of living fungal hyphae is low: assuming a cell volume of $0.17 \mu \mathrm{m}^{3}$ and a density of $0.8 \mathrm{~g} \mathrm{~cm}^{-3}$ (Van Veen and Paul, 1979), the increase in Collimonas biomass in 2 weeks of incubation was calculated to be $0.05 \mu \mathrm{g}$ per gram soil. Ergosterol data were used to calculate the fungal biomass. For zygomycetes, a conversion factor of $3 \mathrm{mg}$ ergosterol per gram biomass can be applied (Olsson et al., 2003). This implies that the amount of $0.5 \mu \mathrm{g}$ of ergosterol that was measured per gram soil is equivalent to $167 \mu \mathrm{g}$ Absidia biomass. This amount of fungal biomass is $3.3 \times 10^{3}$ times bigger than the $0.05 \mu \mathrm{g}$ of Collimonas biomass produced. So even when we assume a low growth efficiency of the collimonads, the reduction of fungal biomass production by collimonads appears to be negligible. Our observation that biomass production by Absidia was not significantly reduced by the presence of collimonads is in line with these calculations. Real-time quantification of $18 \mathrm{~S}$ rRNA fragments of Absidia showed even a trend toward an increase in the presence of collimonads, but this trend was not observed with ergosterol measurements. Overall, it can be concluded that the impact of mycophagous collimonads on fungi is small with respect to biomass production, but the bacteria may be able to introduce morphological changes, for example increased branching. Changes in fungal morphology as a result of the presence of collimonads have been observed by Deveau et al. (2007). They showed that $C$. fungivorans Ter 331 decreased the mycelial extension of $L$. bicolor $S 238 \mathrm{~N}$ but increased the branching density.

The growth dynamics of the mycophagous Trichoderma spp. was examined in a similar way as that of collimonads. Except for the site 22, the results of the real-time PCR analyses indicated that copy numbers of Trichoderma ITS fragments had increased significantly after 2 weeks of exposure to 

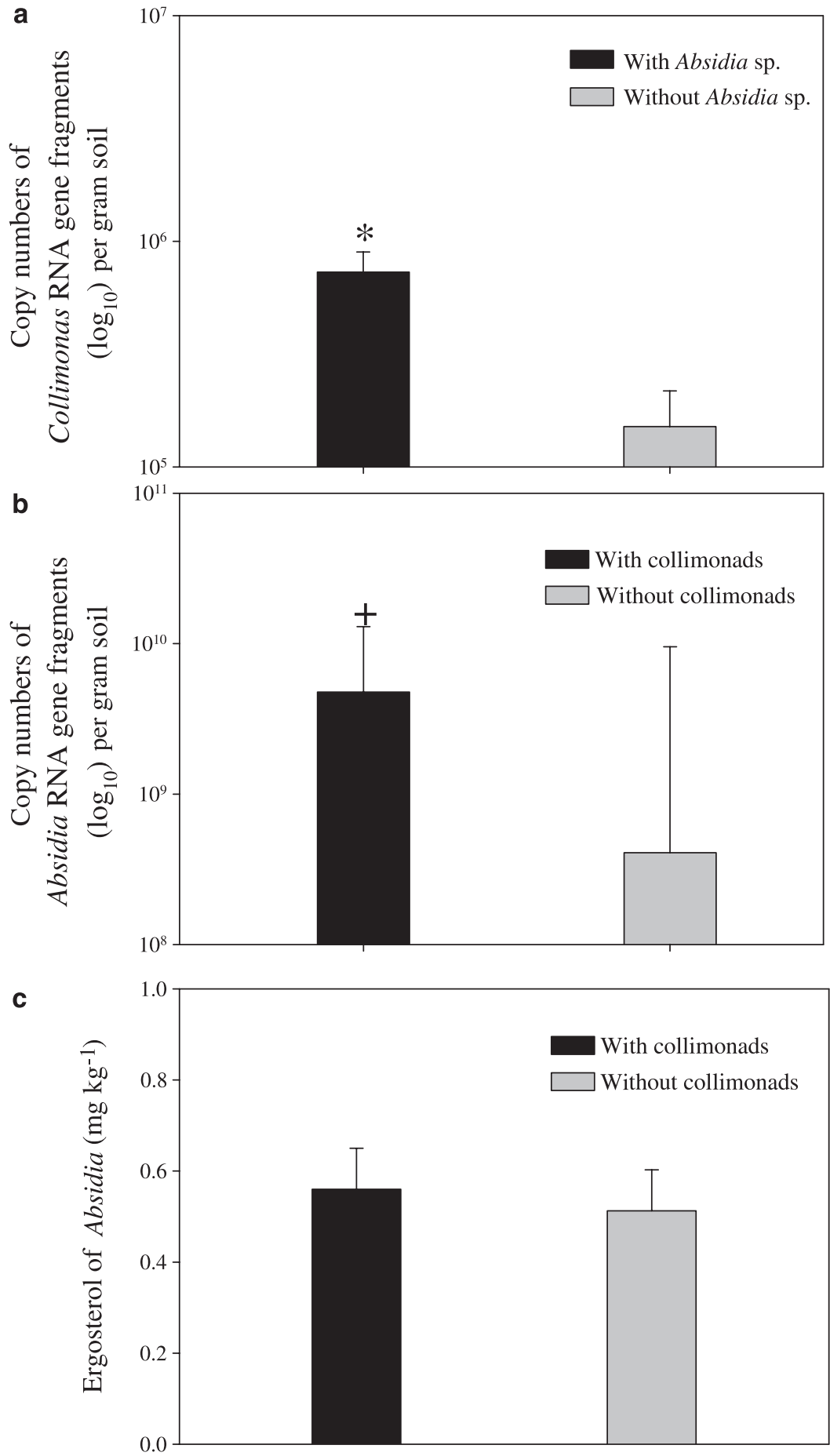

Figure 2 Growth response of collimonads and the fungus Absidia to each other's presence in microcosms containing purified sand. Collimonads were mixed into the soil, and Absidia invaded the soil from a nutrient-rich agar disk. (a) The numbers of 16S rRNA fragments of collimonads, (b) the numbers of 18S rRNA fragments of Absidia and (c) concentration of the fungal membrane component ergosterol. Data represent the means and s.d. of six replicates that were harvested 2 and 3 weeks after the introduction of the Absidia inoculum. Different symbols within a graph indicate significant differences between single (Absidia or collimonads) and mixed (Absidia and collimonads) treatments $\left({ }^{+} P \leqslant 0.1\right.$ and $\left.{ }^{*} P \leqslant 0.05\right)$ based on Tukey's HSD test.

invasion by Absidia hyphae. Thus, the increase of indigenous mycophagous collimonads did not prevent an increased abundance of mycophagous Trichoderma spp. and vice versa. Yet, the experi- ments in the gnotobiotic microcosms containing both collimonads and T. harzianum indicated that the presence of collimonads can have a negative effect on mycophagous growth of Trichoderma spp. 


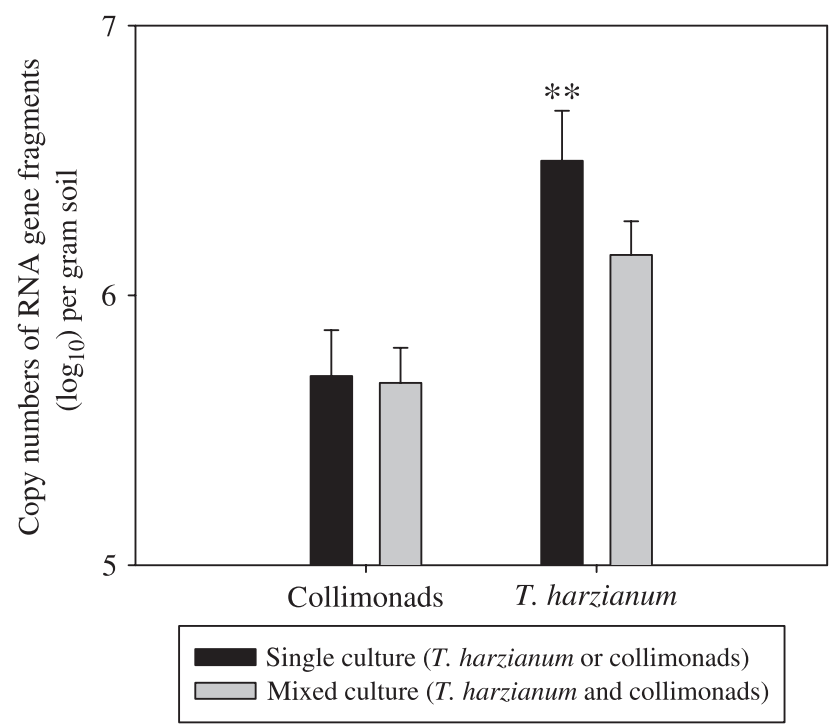

Figure 3 Growth response of collimonads and Trichoderma harzianum to invading hyphae of the fungus Absidia in microcosms containing purified sand. Treatments involved the presence of only collimonads, of only $T$. harzianum or of both collimonads and T. harzianum. Copy numbers of the Collimonas 16S rRNA or Trichoderma internal transcribed spacer (ITS) region were determined by real-time PCR in the mycelial zone of Absidia 3 weeks after the introduction of this fungus. Data represent the means and s.d. for six replicates that were harvested. Different symbols within a graph indicate significant differences between single ( $T$. harzianum or collimonads) and mixed (T. harzianum and collimonads) treatments $\left({ }^{*} P \leqslant 0.01\right)$ based on Tukey's HSD test.

(Figure 3). This negative effect is most likely caused by antibiosis, as resource competition, that is competition for Absidia hyphae, is not likely given the fact that collimonads do not reduce the fungal biomass of Absidia (Figure 2). Another explanation might be competition for the preferential zones to attack Absidia hyphae. However, whereas the attack by collimonads is probably limited to hyphal tips (De Boer et al., 2001), this is not the case for Trichoderma spp., which can penetrate mature hyphae by the appressorium formation (Inbar and Chet, 1994). There is also the possibility that collimonads grew at the expense of $T$. harzianum hyphae, but tests on water agar indicated that this is not likely (data not shown). Production of antibiotics by collimonads has been indicated as an important factor in mycophagous behavior, but it may also result in the inhibition of the biomass formation of Trichoderma (Höppener-Ogawa et al., 2007).

In conclusion, we showed that indigenous soil collimonads respond to the introduction of Absidia hyphae into soil. This supports the importance of mycophagy as a life history characteristic of collimonads. The mycophagous growth of collimonads is of minor importance for fungal biomass dynamics but may affect fungal growth patterns and also community dynamics. In addition, we showed that collimonads can have a negative effect on the mycophagous growth of T. harzianum.

\section{Acknowledgements}

This is publication no. 4411 NIOO-KNAW of the Netherlands Institute of Ecology. The funding of the research of Sachie Höppener-Ogawa by the Netherlands Organization for Scientific Research (NWO) is highly appreciated.

\section{References}

De Boer W, Klein Gunnewiek PJA, Kowalchuk GA, van Veen JA. (2001). Growth of chitinolytic dune soil betasubclass Proteobacteria in response to invading fungal hyphae. Appl Environ Microbiol 67: 3358-3362.

De Boer W, Folman LB, Summerbell RC, Boddy L. (2005). Living in a fungal world: impact of fungi on soil bacterial niche development. FEMS Microbiol Rev 29: 795-811.

De Boer W, Wagenaar AM, Klein Gunnewiek PJA, van Veen JA. (2007). In vitro suppression of fungi caused by combinations of apparently non-antagonistic soil bacteria. FEMS Microbiol Ecol 59: 177-185.

De Boer W, Klein Gunnewiek PJA, Lafeber P, Janse JD, Spit BE, Woldendorp JW. (1998). Anti-fungal properties of chitinolytic dune soil bacteria. Soil Biol Biochem 30: 193-203.

De Boer W, Leveau JHJ, Kowalchuk GA, Klein Gunnewiek PJA, Abeln ECA, Figge MJ et al. (2004). Collimonas fungivorans gen. nov., sp nov., a chitinolytic soil bacterium with the ability to grow on living fungal hyphae. Int J Syst Evol Microbiol 54: 857-864.

De Ridder-Duine AS, Smant W, van der Wal A, van Veen JA, De Boer W. (2006). Evaluation of a simple, nonalkaline extraction protocol to quantify soil ergosterol. Pedobiologia 50: 293-300.

Deveau A, Palin B, Delaruelle C, Peter M, Kohler A, Pierrat JC et al. (2007). The mycorrhiza helper Pseudomonas fluorescens BBc6R8 has a specific priming effect on the growth, morphology and gene expression of the ectomycorrhizal fungus Laccaria bicolor S238N. New Phytol 175: 743-755.

Donzelli BGG, Harman GE. (2001). Interaction of ammonium, glucose, and chitin regulates the expression of cell wall-degrading enzymes in Trichoderma atroviride strain P1. Appl Environ Microb 67: 5643-5647.

Elad Y, Chet I, Boyle P, Henis Y. (1983). Parasitism of Trichoderma-spp on Rhizoctonia-solani and Sclerotium-rolfsii scanning electron-microscopy and fluorescence microscopy. Phytopathology 73: 85-88.

Gardes M, Bruns TD. (1993). ITS primers with enhanced specificity for basidiomycetes-application to the identification of mycorrhizae and rusts. Mol Ecol 2: 113-118.

Hagn A, Wallisch S, Radl V, Munch JC, Schloter M. (2007). A new cultivation independent approach to detect and monitor common Trichoderma species in soils. $J$ Microbiol Meth 69: 86-92.

Harman GE. (2006). Overview of mechanisms and uses of Trichoderma spp. Phytopathology 96: 190-194.

Hermosa MR, Grondona I, Iturriaga EA, Diaz-Minguez JM, Castro C, Monte E et al. (2000). Molecular characterization and identification of biocontrol isolates of Trichoderma spp. Appl Environ Microbiol 66: 1890-1898. 
Heuer H, Krsek M, Baker P, Smalla K, Wellington EMH. (1997). Analysis of actinomycete communities by specific amplification of genes encoding 16S rRNA and gel-electrophoretic separation in denaturing gradients. Appl Environ Microbiol 63: 3233-3241.

Hoffmann K, Discher S, Voigt K. (2007). Revision of the genus Absidia (Mucorales, Zygomycetes) based on physiological, phylogenetic, and morphological characters; thermotolerant Absidia spp. form a coherent group, Mycocladiaceae fam. nov. Mycol Res 111: 1169-1183.

Höppener-Ogawa S, Leveau JHJ, Smant W, van Veen JA, de Boer W. (2007). Specific detection and real-time PCR quantification of potentially mycophagous bacteria belonging to the genus Collimonas in different soil ecosystems. Appl Environ Microbiol 73: 4191-4197.

Höppener-Ogawa S, de Boer W, Leveau JH, Veen JA, Brandt E, Vanlaere E et al. (2008). Collimonas arenae sp. nov. and Collimonas pratensis sp. nov., isolated from (semi-) natural grassland soils. Int J Syst Evol Microbiol 58: 414-419.

Inbar J, Chet I. (1994). A newly isolated lectin from the plant pathogenic fungus Sclerotium rolfsii: purification, characterization and role in mycoparasitism. Microbiology 140: 651-657.

Izumi H, Cairney JWG, Killham K, Moore E, Alexander IJ, Anderson IC. (2008). Bacteria associated with ectomycorrhizas of slash pine (Pinus elliottii) in Southeastern Queensland, Australia. FEMS Microbiol Lett 282: 196-204.

Kowalchuk GA, De Bruijn FJ, Head IM, Akkermans ADJ, van Elsas JD. (2004). Molecular Microbial Ecology Manual, 2nd edn, vol. 1. Kluwer Academic Publishers.

Legendre P, Anderson MJ. (1999). Distance-based redundancy analysis: testing multispecies responses in multifactorial ecological experiments. Ecol Monogr 69: 1-24.

Leveau JHJ, Preston GM. (2008). Bacterial mycophagy: definition and diagnosis of a unique bacterial-fungal interaction. New Phytol 177: 859-876.

Lorito M, Woo SL, Fernandez IG, Colucci G, Harman GE, Pintor-Toro JA et al. (1998). Genes from mycoparasitic fungi as a source for improving plant resistance to fungal pathogens. Proc Natl Acad Sci USA 95: 7860-7865.

Olsson PA, Larsson L, Bago B, Wallander H, van Aarle IM. (2003). Ergosterol and fatty acids for biomass estimation of mycorrhizal fungi. New Phytol 159: 7-10.
Rubio MB, Hermosa MR, Keck E, Monte E. (2005). Specific PCR assays for the detection and quantification of DNA from the biocontrol strain Trichoderma harzianum 2413 in soil. Microb Ecol 49: 25-33.

Salles JF, De Souza FA, van Elsas JD. (2002). Molecular method to assess the diversity of Burkholderia species in environmental samples. Appl Environ Microbiol 68: 1595-1603.

Samuels GJ. (1996). Trichoderma: a review of biology and systematics of the genus. Mycol Res 100: 923-935.

Singh T, Arora DK. (2001). Motility and chemotactic response of Pseudomonas fluorescens toward chemoattractants present in the exudate of Macrophomina phaseolina. Microbiol Res 156: 343-351.

Taylor DL, Bruns TD. (1999). Community structure of ectomycorrhizal fungi in a Pinus muricata forest: minimal overlap between the mature forest and resistant propagule communities. Mol Ecol 8: 1837-1850.

Ter Braak CJF, Šmilauer P. (2002). CANOCO Reference Manual and CanoDraw for Windows User's Guide: Software for Canonical Community Ordination (version 4.5). Microcomputer Power: Ithaca, NY.

Vainio EJ, Hantula J. (2000). Direct analysis of woodinhabiting fungi using denaturing gradient gel electrophoresis of amplified ribosomal DNA. Mycol Res 104: 927-936.

Van Veen JA, Paul EA. (1979). Conversion of biovolume measurements of soil organisms, grown under various moisture tensions, to biomass and their nutrient content. Appl Environ Microbiol 37: 686-692.

Whipps JM. (2001). Microbial interactions and biocontrol in the rhizosphere. J Exp Bot 52: 487-511.

White TJ. (1990). PCR Protocols: A Guide to Methods and Applications. Academic press inc., San Diego, pp 315-322.

Widmer F, Seidler RJ, Gillevet PM, Watrud LS, Di Giovanni GD. (1998). A highly selective PCR protocol for detecting 16S rRNA genes of the genus Pseudomonas (sensu stricto) in environmental samples. Appl Environ Microbiol 64: 2545-2553.

Yergeau E, Bokhorst S, Huiskes AHL, Boschker HTS, Aerts R, Kowalchuk GA. (2007). Size and structure of bacterial, fungal and nematode communities along an Antarctic environmental gradient. FEMS Microbiol Ecol 59: 436-451.

Supplementary Information accompanies the paper on The ISME Journal website (http://www.nature.com/ismej) 